MRS Advances (C) 2016 Materials Research Society

DOI: $10.1557 /$ adv.2016.540

\title{
Skin Hydration Sensor for Customizable Electronic Textiles
}

\author{
Murat A. Yokus ${ }^{1,2}$ and Michael A. Daniele $e^{1,3}$ \\ ${ }^{1}$ Department of Electrical and Computer Engineering, North Carolina State University, \\ Raleigh, NC 27606, U.S.A. \\ ${ }^{2}$ Department of Textile Engineering, Chemistry \& Science, North Carolina State University, \\ Raleigh, NC 27606, U.S.A. \\ 3 Joint Department of Biomedical Engineering, North Carolina State University and \\ University of North Carolina, Chapel Hill, 911 Oval Dr., Raleigh, NC 27695, U.S.A.
}

\begin{abstract}
This paper introduces the design and simulated operation of a capacitive hydration sensor for integration into textile-based electronics. The multilayer patch is composed of a textile layer and an attached series of serpentine-interdigitated electrodes. The model used for simulations incorporated this design onto a representative model of skin. The serpentine-interdigitated electrodes are electrodes for capacitive measurement of skin hydration. In this study, the capacitance change relative to skin hydration was simulated using finite element analysis. The simulation results suggest the fabric layer had little effect on the capacitance of the sensor. Furthermore, the frequency domain simulations indicated that the capacitance of the sensor decreased with increasing frequency, and the decrease in capacitance was more significant for the dry skin compared to the wet skin. Therefore, the variation in the capacitance value of the serpentine-interdigitated electrodes can be employed for continuous skin hydration detection.
\end{abstract}

\section{INTRODUCTION}

Hydration plays a significant role in cellular homeostasis and thermoregulation. The human body expels water mainly by discharging urine from kidneys, excreting sweat via the skin and exhaling water vapor from the lungs. Water loss from sweating can be between $0.3 \mathrm{~L} / \mathrm{h}-0.6 \mathrm{~L} / \mathrm{h}$, depending on environmental conditions and activity levels. Water loss, as low as $2 \%$ of the body mass, leads to dehydration which manifests as loss in physical and cognitive performance, and alterations in kidney, heart and gastrointestinal functions [1]. Therefore, monitoring for dehydration is critical for the determination of physiological status of people experiencing significant heat stress or performing strenuous activity, such as emergency operators, athletes, and military personnel.

Electrical methods are the most commonly used techniques for evaluation of skin hydration. Commercial products, such as Skicon ${ }^{\circledR}$ and Corneometer ${ }^{\circledR}$, measure skin hydration based on the change in skin conductance and capacitance, respectively [2]. There are at least three major factors affecting the accuracy of measuring skin conductance including dryness, ion concentration and measurement frequency [3]. Electron conduction dominates in dry skin; however, conduction due to exchange of protons on hydrogen bonded water molecules dominates in hydrated skin and is a function of water content. Nonetheless, the measurement of skin conductivity may show significant variation dependent on skin surface (e.g. hair, oil) and skin temperature. Similarly, skin capacitance may change with distribution of water, ions and 
dipoles in the skin, which directly affects the electrical field penetration into skin [3]. However, capacitive measurement techniques are advantageous over skin conductance measurements because they mitigate surface effects by measuring larger and deeper tissue volumes (i.e. up to $40 \mu \mathrm{m}$ in depth) [2].

Alternatives to electrical methods for measuring skin hydration have been developed. Radio frequency absorptiometry was used to monitor hydration by relating electromagnetic field absorption and reflection parameters to the total weight loss due to sweating [4]. However, location based RF transmission variation was exhibited due to the change in tissue composition across the skin surface. Optical sweat sensors have been explored which utilize a colorimetric detection scheme to quantify ion concentration in sweat and subsequently infer hydration. [5]. A near-infrared (NIR) imaging method was investigated to measure NIR light absorption intensity as a function of concentration of water in the skin [6]. Although promising such alternative methods for measuring skin hydration are not yet suitable for integration into wearable sensor systems due to the operational and power requirements of optical systems, or the substantial response variation under mechanical deformation or movement across the skin's surface.

Consequently, many wearable hydration sensors have focused on the miniaturization of electronic systems. Impedance [7], capacitance [8], and resonance [9] based stretchable epidermal sensors are some of the recent techniques utilized in hydration monitoring. Also, wrist-worn wearable sensor arrays [10] and "tattoo-like" epidermal devices [11] have recently been reported which measure the metabolites and electrolytes in sweat which can denote hydration level.

Accordingly, we have chosen to begin investigation into a simple capacitive hydration sensor that can be easily integrated with textile-based systems. Herein, a capacitive hydration sensor for integration with textile-based system is designed and simulated. The capacitive hydration sensor and measurement concept are illustrated in Figure 1.

a)

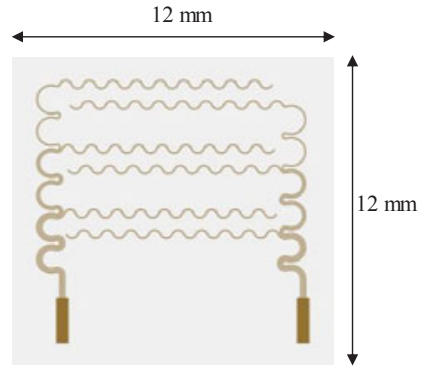

b)

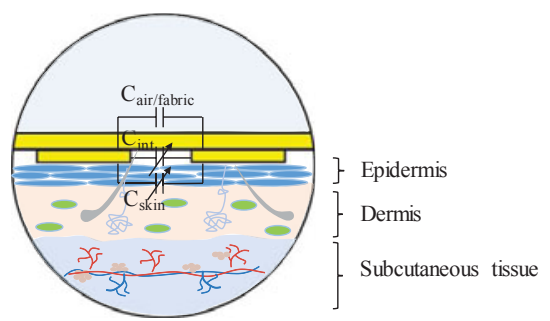

Figure 1. a) Design of capacitance hydration sensor with serpentine-interdigitated electrodes. Electrodes have a width of $50 \mu \mathrm{m}$ and separation of $50 \mu \mathrm{m}$. A $300 \mathrm{~nm}$ gold layer was used as the model for the electrodes. b) Cross-sectional view of the capacitive sensor. $\mathrm{C}_{\text {air/fabric }}, \mathrm{C}_{\text {int }}$, and $\mathrm{C}_{\text {skin }}$ are the capacitances of air/fabric, intermediate layer and underlying skin, respectively.

The proposed capacitive hydration sensor utilizes serpentine-interdigitated electrodes (width of $50 \mu \mathrm{m})$. The selected width of the electrodes $(50 \mu \mathrm{m})$ provides for flexibility and conformal adherence to the skin and textile interfaces; moreover, these electrode dimensions can be easily 
fabricated with current photolithography techniques. The serpentine design of the electrodes accommodates for mechanical deformation experienced during use [12]. Increased mechanical compliance of the electrodes is advantageous for integration of the sensor on a flexible and stretchable textile system. Electrodes are encapsulated in polyimide $(1 \mu \mathrm{m}$ top and $1 \mu \mathrm{m}$ bottom, $\left.\varepsilon_{\mathrm{r}}=3.4\right)$ to improve endurance under cyclic tensile loading. Only the individual electrodes are encapsulated, which provides for a minimally occlusive patch when applied to the textile or skin. The serpentine-shaped electrodes can be easily integrated onto wearables such as on a knit fabric to realize a wearable or skin mounted patches. In the model, textile lies at the top of the multilayer structure and facilitates the air and vapor permeability to the skin. Fig. 1(b) illustrates the capacitive sensing mechanism of the patch, where $\mathrm{C}_{\text {air/abric }}, \mathrm{C}_{\mathrm{int}}$, and $\mathrm{C}_{\text {skin }}$ are capacitances due to air/fabric, intermediate layer and underlying skin. The skin capacitance $\left(\mathrm{C}_{\text {skin }}\right)$ and the intermediate layer capacitance $\left(\mathrm{C}_{\mathrm{int}}\right)$ vary with hydration of the skin as the dielectric constant of the medium changes; therefore, the capacitive sensor patch could give a feedback to user regarding hydration.

\section{EXPERIMENTAL}

The operation of the capacitive hydration sensor was modeled in COMSOL Multiphysics ${ }^{\circledR}$ software. The 3D simulations were performed with Electrostatics and Electric Current Models. Under static conditions the Electrostatic Model solves Maxwell's equations, Eqn. 1-2, for electric potential, where $\mathrm{D}, \rho_{\mathrm{v}}, \mathrm{E}$, and $V$ are electric flux density $\left(\mathrm{C} / \mathrm{m}^{2}\right)$, electric charge density $\left(\mathrm{C} / \mathrm{m}^{3}\right)$, electric field intensity $(\mathrm{V} / \mathrm{m})$, and electric potential $(\mathrm{V})$, respectively [17]. Divergence and gradient operators are shown as $(\nabla \cdot)$ and $(\nabla)$.

$$
\begin{aligned}
& \nabla \cdot \mathbf{D}=\rho_{\mathrm{v}} \\
& \mathbf{E}=-\nabla V
\end{aligned}
$$

Similarly, the Electric Current Model utilizes the Maxwell's equations in Eqn. 2-4 in frequency domain, where $\mathrm{J}, \mathrm{Q}_{\mathrm{j}}, \sigma, \mathrm{J}_{\mathrm{e}}$ are current density $\left(\mathrm{A} / \mathrm{m}^{2}\right)$, current source $\left(\mathrm{A} / \mathrm{m}^{3}\right)$, electrical conductivity $(\mathrm{S} / \mathrm{m})$, and externally generated current density $\left(\mathrm{A} / \mathrm{m}^{2}\right)$.

$$
\begin{aligned}
& \nabla \cdot \mathbf{J}=\mathrm{Q}_{\mathrm{j}} \\
& \mathbf{J}=\sigma \mathbf{E}+\mathrm{j} \omega \mathbf{D}+\mathbf{J}_{\mathbf{e}}
\end{aligned}
$$

The Stationary Electrostatic Model was used to compute the electric field, potential distribution and capacitance; whereas, the Electric Current Model was utilized to compute the value of capacitance in frequency-domain from $10 \mathrm{~Hz}$ to $0.1 \mathrm{MHz}$. The applied voltage amplitude was kept constant for both simulations $(1 \mathrm{~V})$. The relative tolerance for the stationary and frequencydomain studies was set to 0.001 . Partitioned boundary boxes (width: $20 \mathrm{~mm}$, depth: $20 \mathrm{~mm}$, height: $20 \mathrm{~mm}$ ) were used at the top and bottom of the patch to model the dielectric properties of air, fabric, and skin. To simulate the effect of the fabric on top of the capacitive sensor, a dielectric permittivity sweep from 1 to 10 was performed in the Stationary Electrostatic Model and the corresponding capacitance values were calculated.

\section{DISCUSSION}


The capacitance of interdigitated structure as a function of its geometrical parameters has been reported $[13,14]$; hence, the proposed geometry (Fig. 1a) was selected as a practical design for both simulation and future fabrication. As reported by Wang [13], the capacitance of the interdigitated electrodes is proportional to an increase in electrode thickness, electrode width, electrode overlap, number of electrodes, and dielectric constant of the medium, and inversely proportional to electrode spacing.

The capacitance of the sensor with respect to the change of the top dielectric layer's relative permittivity $\left(\varepsilon_{\mathrm{r}}\right)$ value is shown in Figure 2a. The capacitance was calculated by incorporating the relative permittivity of the dry and wet skin into the model, which were reported for $10 \mathrm{~Hz}[15$, 16]. In the model, it is assumed that the relative permittivity of the skin does not change significantly from $10 \mathrm{~Hz}$ to DC. The sensor on the wet skin showed a larger capacitance compared to the dry skin. This was attributed to the change in the dielectric constant of the skin with hydration. The capacitance in the air medium $\left(\varepsilon_{\mathrm{r}}=1\right)$ on dry skin was computed as $29.3 \mathrm{pF}$. The dielectric constants of commonly used textiles (e.g. polyester, polyamide etc.) fall in the range of the hatch area in Fig. 2(a). Addition of a textile layer $\left(\varepsilon_{\mathrm{r}}=4\right)$ on top of the capacitive sensor in the model increased the capacitance by 1.2 and $1.8 \mathrm{pF}$ for dry and wet skin, respectively. It is important to note that the textile layer is not modelled as a porous media. Future models may incorporate the significant porosity of advanced textiles, which would decrease the effective permittivity of the medium, resulting in a decrease in total capacitance. Further increases in the permittivity of the textile layer increased the capacitance value for both wet and dry skin models. This could be an indication of absorbency and wetting effects of the textile under perspiring conditions.

The electric field and potential distribution of the capacitive sensor are shown in Fig. 2(b) and (d), respectively. There was a strong capacitive coupling between the serpentine inner interdigitated fingers separated by 0.4 and $1.5 \mathrm{~mm}$ in Fig. 2(b). Fig. 2(c) shows the crosssectional potential distribution of the capacitive sensor on the dry skin with a textile layer $\left(\varepsilon_{\mathrm{r}}=2\right)$. The cross-sectional image was obtained by using a vertical cut plane in Fig. 1(a). It was shown that the field penetrated more into the textile and dielectric layer than the skin due to the higher dielectric constant of the skin relative to the air.
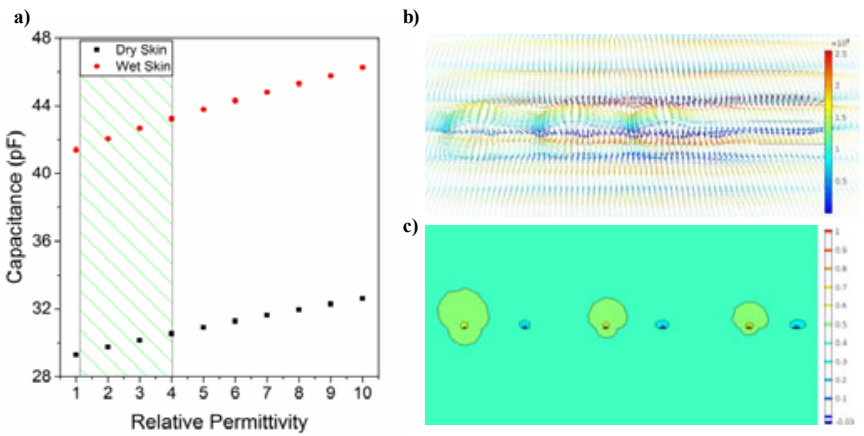

Figure 2. a) Capacitance change as a function of the textile layer's relative permittivity for dry and wet skin models. The hatch area indicates the relative permittivity range for common textile 
materials. b) Side view electric field distribution of capacitive sensor on the dry skin with a fabric layer $\left(\varepsilon_{\mathrm{r}}=2\right)$. c) Cross-sectional view of electrical potential distribution of the serpentine capacitive sensor on the dry skin.

The capacitance change as a function of frequency is shown in Figure 3. The equivalent circuitry of the capacitive sensor can be written as parallel combination of a capacitor and a resistor. The complex admittance of the RC circuit is given as in Eqn. 5, where Y, G, $\omega$, and C are used for admittance, conductance, radial frequency, and capacitance.

$$
Y=G+j \omega C
$$

The admittance of the capacitive hydration sensor was calculated. Subsequently, the capacitance value of the serpentine-interdigitated electrodes was calculated from the imaginary part of the admittance. The decrease in the dielectric constant and the increase in the conductivity with increasing frequency (i.e. the dispersion effects), which corresponds to previous reports $[15,16]$, were included in the model. The simulation determined the capacitance of the sensor decreased with increasing frequency for the dry and wet skin. This is a result, in part, of the decrease in the dielectric constant with respect to increasing frequency. The decrease in the capacitance also may be attributed to the increasing electrical conductivity of the skin with respect to increasing frequency, which results in an increase in the real part (i.e. conductance) of the admittance. The capacitance decreases as radial frequency $(\omega)$ increases, and the decrease in the capacitance was much smaller for the wet skin model with increasing frequency. Ultimately, the capacitive response of the serpentine-interdigitated electrodes at high frequencies can be used to assess skin hydration.

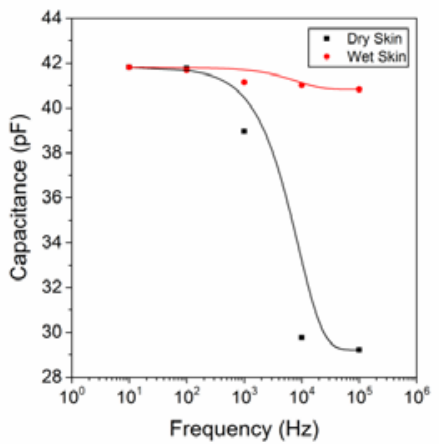

Figure 3. Capacitance change as a function of frequency for dry and wet skin models.

\section{CONCLUSIONS}

Design and simulation of a capacitive sensor were studied for potential use in skin hydration or sweat monitors to be integrated onto textile systems. The capacitance change of the serpentine-interdigitated electrodes was modeled as a device at the skin-textile interface. The 
electrical properties of the sensor were simulated with models of hydrated and dehydrated skin. The capacitance of the sensor increased as a function of skin hydration. We also note that the addition of the textile layer to the model did not raise the capacitance significantly. The simulation results also suggest that higher frequencies are more sensitive to changes in hydration.

Continued modeling efforts will explore the change in capacitance with dynamic conditions, where the sensor itself undergoes mechanical stress and deformation as a result of a wearer's movements. Future experimental work will include fabrication, in vitro characterization (linearity, response time, and sensitivity) and in vivo operation of the capacitive hydration sensor integrated into a textile system.

\section{ACKNOWLEDGMENTS}

This work was supported by the U.S. National Science Foundation through the Nanosystems Engineering Research Center for Advanced Self-Powered Systems of Integrated Sensors and Technologies (ASSIST) under Grant EEC 1160483.

\section{REFERENCES}

${ }^{1}$ B.M. Popkin, K.E. D’Anci, and I.H. Rosenberg, Nutr. Rev. 68, 439 (2010).

${ }^{2}$ P. Clarys, R. Clijsen, J. Taeymans, and A.O. Barel, Skin Res. Technol. Off. J. Int. Soc. Bioeng. Skin ISBS Int. Soc. Digit. Imaging Skin ISDIS Int. Soc. Skin Imaging ISSI 18, 316 (2012).

${ }^{3}$ E. Berardesca and European Group for Efficacy Measurements on Cosmetics and Other Topical Products (EEMCO), Skin Res. Technol. 3, 126 (1997).

${ }^{4}$ D.S. Moran, Y. Heled, M. Margaliot, Y. Shani, A. Laor, S. Margaliot, E.E. Bickels, and Y. Shapiro, Physiol. Meas. 25, 51 (2004).

${ }^{5}$ Al-omari Mahmoud, G. Liu, A. Mueller, A. Mock, R.N. Ghosh, K. Smith, and T. Kaya, J. Appl. Phys. 116, 183102 (2014).

${ }^{6}$ S.L. Zhang, C.L. Meyers, K. Subramanyan, and T.M. Hancewicz, J. Biomed. Opt. 10, 031107 (2005).

${ }^{7}$ X. Huang, W.-H. Yeo, Y. Liu, and J.A. Rogers, Biointerphases 7, 1 (2012).

${ }^{8}$ P. Wei, B. Morey, T. Dyson, N. McMahon, Y.-Y. Hsu, S. Gazman, L. Klinker, B. Ives, K. Dowling, and C. Rafferty, in 2013 IEEE Sens. (2013), pp. 1-4.

${ }^{9}$ X. Huang, Y. Liu, H. Cheng, W.-J. Shin, J.A. Fan, Z. Liu, C.-J. Lu, G.-W. Kong, K. Chen, D. Patnaik, S.-H. Lee, S. Hage-Ali, Y. Huang, and J.A. Rogers, Adv. Funct. Mater. 24, 3846 (2014).

${ }^{10}$ W. Gao, S. Emaminejad, H.Y.Y. Nyein, S. Challa, K. Chen, A. Peck, H.M. Fahad, H. Ota, H. Shiraki, D. Kiriya, D.-H. Lien, G.A. Brooks, R.W. Davis, and A. Javey, Nature 529, 509 (2016).

${ }^{11}$ A.J. Bandodkar, D. Molinnus, O. Mirza, T. Guinovart, J.R. Windmiller, G. Valdés-Ramírez, F.J. Andrade, M.J. Schöning, and J. Wang, Biosens. Bioelectron. 54, 603 (2014).

${ }^{12}$ D.S. Gray, J. Tien, and C.S. Chen, Adv. Mater. 16, 393 (2004).

${ }^{13}$ Y. Wang, N. Chong, Y.L. Cheng, H.L.W. Chan, and C.L. Choy, Microelectron. Eng. 66, 880 (2003).

${ }^{14}$ S.S. Gevorgian, T. Martinsson, P.L.J. Linner, and E.L. Kollberg, IEEE Trans. Microw. Theory Tech. 44, 896 (1996).

${ }^{15}$ D. Miklavčič, N. Pavšelj, and F. Hart, Wiley Encyclopedia of Biomedical Engineering. (2006).

${ }^{16}$ S. Gabriel, R.W. Lau, and C. Gabriel, Phys. Med. Biol. 41, 2271 (1996).

${ }^{17}$ COMSOL AC/DC Module User's Guide, Version 5.2. 